\title{
Caracterización de la Política de Proyección Social de la Universidad Católica de Córdoba (UCC) para evaluar el impacto formativo en los graduados
}

\section{Characterization of the Social Projection Policy of the Catholic University of Córdoba (UCC) to evaluate the educational impact}

\author{
Federico Javier Giraudo ${ }^{1}$ \\ 1. Universidad Católica de Córdoba. Facultad de Educación. Doctorado en Educación. \\ Correspondencia: Federico Javier Giraudo. Universidad Católica de Córdoba. Facultad de Educación. Doctorado en Educación. E-mail: fjgiraudo@hotmail.com
}

\section{Resumen}

La Universidad Católica de Córdoba (Argentina) ha implementado una política de proyección social desde el año 2011, la cual forma parte de un objetivo central a nivel institucional: concebir las funciones sustantivas y centrales de la universidad desde la gestión socialmente responsable. La búsqueda de este objetivo tiene su sustento en el paradigma educativo ignaciano, el cual busca incidir desde allí en la formación de los futuros graduados.

Frente a ello, se plantea una investigación que busca indagar sobre el impacto que logra en la formación de los graduados la política de proyección social bajo el enfoque de Responsabilidad Social Universitaria adoptado por la Universidad Católica de Córdoba.

Una etapa esencial de esta investigación es lograr caracterizar la política mencionada a los fines de correlacionar los procesos educativos de acuerdo al paradigma educativo adoptado en la universidad con respecto a la formación y el perfil de sus graduados.

Esta caracterización se realizó a partir de un enfoque analítico interpretativo, retrospectivo y transversal, desarrollado a través de un diseño metodológico cualitativo, basado en análisis documental y entrevistas en profundidad con los referentes institucionales.

Los resultados de caracterización de la PPS permitieron considerar dos categorías de análisis: por un lado, el vínculo de la Universidad con la comunidad de acuerdo a propiedades prestablecidas, y por otro, el impacto de la política sobre los futuros graduados de acuerdo al paradigma educativo adoptado.

Ante la carencia de herramientas adecuadas de evaluación de impacto formativo en los perfiles de los egresados universitarios, esta investigación pretende contribuir con avances instrumentales útiles en la medición de la efectividad de la política de proyección social de la Universidad Católica de Córdoba, los cuales poseen la potencialidad de hacerse extensivos para su empleo en otras universidades confiadas a la Compañía de Jesús, y de la región.

Palabras clave: Caracterización, Política de Proyección Social, Universidad Católica de Córdoba, impacto formativo, graduados.

\section{Abstract}

The Catholic University of Córdoba (Argentina) has implemented a social projection policy since 2011, which is part of a central objective at the institutional level: to conceive the substantive and central functions 
of the university from socially responsible management. The search for this objective is supported by the Ignatian educational paradigm, which seeks to influence the training of future graduates from there.

Faced with this, an investigation is proposed that seeks to investigate the impact that the policy of social projection, under the approach of University Social Responsibility, adopted by the Catholic University of Córdoba achieves in the training of graduates.

An essential stage of this research is to characterise the aforementioned policy in order to correlate the educational processes according to the educational paradigm adopted at the university with respect to the training and profile of its graduates.

This characterisation was carried out from an interpretive, retrospective and cross-sectional analytical approach, developed through a qualitative methodological design, based on documentary analysis and indepth interviews with institutional referents.

The results of the characterisation of the PPS allowed considering two categories of analysis: on the one hand, the link between the University and the community according to pre-established properties, and on the other, the impact of the policy on future graduates according to the adopted educational paradigm.

Given the lack of adequate tools for evaluating the training impact on the profiles of university graduates, this research aims to contribute with useful instrumental advances in measuring the effectiveness of the social projection policy of the Catholic University of Córdoba, which have the potential to be extended for employment in other universities entrusted to the Society of Jesus, and in the region.

Keywords: Characterization, Social Projection Policy, Catholic University of Córdoba, training impact, graduates.

\section{Introducción}

La proyección social es una función universitaria de transferencia de conocimientos a la sociedad que en la Universidad Católica de Córdoba (UCC) se sustenta en un modelo educativo en el cual los procesos de transmisión (enseñanza y aprendizaje) y producción del conocimiento (investigación) se orientan a que estudiantes y docentes se involucren y contribuyan al desarrollo humano integral de la comunidad en la que la universidad se encuentra inserta, propiciando su implicación en todas las actividades humanas en las que pueda hacer su contribución; promueve un modelo académico asociativo y multidisciplinario, comprometido con la creación e intercambio de conocimientos en íntima relación con los diferentes actores sociales, $y$ en el que las funciones de docencia, investigación, proyección social y gestión interna estén integradas.

La UCC, perteneciente a la Asociación de Universidades Confiadas a la Compañía de Jesús en América Latina (AUSJAL) conformada por 31 universidades jesuitas de Latinoamérica, ha institucionalizado una política de proyección social (PPS), entendiendo a esta como el proceso de participación sistemática y activa de la Universidad en el desarrollo humano sustentable de la comunidad de la que forma parte, (...) propiciando la co-producción entre distintos actores y saberes hacia un conocimiento de calidad y pertinencia en términos de asociación, a fin de promover el progreso, crear capital social, vincular la educación de los estudiantes con la realidad exterior, y funcionar de interlocutor en la solución de los problemas ${ }^{1}$.

En este sentido, en la Resolución Rectoral N. ${ }^{\circ}$ $1092 / 2011^{2}$ que contiene la política relativa a la proyección social de la UCC, se adopta el término de proyección social a fin de enfatizar la necesidad y la opción institucional de:

a. Concebir esta función universitaria (proyección social) en términos de desarrollo, evitando que las relaciones entre la universidad y la comunidad exterior sean solo en carácter de donación, filantropía o ayuda unilateral, de modo de propiciar la asociación y la creación de comunidades de aprendizaje mutuo, en las cuales los socios externos también ayuden a la universidad a cumplir con su rol académico y educativo.

b. Descentralizar los procesos de enseñanza y aprendizaje desde el claustro universitario a la sociedad, fomentando nuevas dinámicas de aprendizaje y transmisión de conocimiento (docencia), generación de conocimiento (investigación) y formación humana significativa (experiencia vivencial y reflexión crítica) con participación de actores externos.

c. Hacer manifiesta la opción fundamental por los sectores desfavorecidos de la sociedad, en coherencia con la misión de la Compañía de Jesús definida como el servicio de la fe y la promoción de la justicia. 
d. Denotar la intención de producir un impacto beneficioso en la sociedad a través de acciones responsables y altamente calificadas propias de las capacidades académicas y profesionales de la universidad.

Esta concepción de la proyección social se encuentra vinculada al paradigma educativo llevado adelante en las instituciones educativas confiadas a la Compañía de Jesús en el mundo, a través del cual no sólo se persigue el objetivo de formar personas competentes para el ejercicio de la profesión, sino una formación integral que aporte herramientas para la transformación de la realidad de acuerdo a la concepción buscada a través de la pedagogía ignaciana.

Desde esta mirada centrada en la formación integral, definida en el paradigma educativo de las instituciones confiadas a la Compañía de Jesús, queda manifiesto que la transformación social es consecuencia de la acción de numerosos actores entre los cuales se encuentran los graduados universitarios. La tarea de formar profesionales sensibles a la realidad y lúcidos para transformarla transciende lo meramente disciplinar y la formación técnica. Implica instrumentar de manera cuidadosa una política de formación que posibilite desarrollar la sensibilidad hacia el entorno, especialmente dirigida hacia los sectores más desfavorecidos, para que los graduados sean en su esencia conscientes, competentes, comprometidos y compasivos ${ }^{3}$.

Es así que la transformación social dependerá en parte, de la previa transformación de los futuros profesionales, objetivo primordial de la política de proyección social que implementa la UCC.

Ahora bien, para responder al interrogante de qué impacto logra en la formación de los graduados la PPS bajo el enfoque de Responsabilidad Social Universitaria adoptado por la Universidad Católica de Córdoba, es necesario caracterizar esta política delimitando los efectos deseados en los graduados a través de su implementación siguiendo los objetivos enunciados y las líneas operativas de concreción.

\section{Materiales y métodos}

Para el presente trabajo de investigación se siguió un enfoque analítico interpretativo, retrospectivo y transversal, desarrollado a través de un diseño metodológico cualitativo. Para lograr el análisis de caracterización de la PPS, el trabajo de investigación se realizó en dos etapas:

a. En primera instancia, se indagó en profundidad a través de un análisis documental, el paradigma educativo, la formación y pedagogía en las instituciones de la Compañía de Jesús, así como sus fundamentos y estrategias de implementación de la PPS adoptada a los fines de delimitar el alcance de esta política en el proceso formativo y el impacto que se busca lograr en los graduados. Las fuentes consultadas fueron numerosas, dentro de las cuales se destacan especialmente los discursos y documentos escritos por el Prepósito General de la Compañía de Jesús P. Peter Hans Kolvenbach S.J. ${ }^{4-10}$, el Decreto 4 de la Congregación General 32 $2^{11}$, los aportes del Padre Pedro Arrupe S.J. ${ }^{12,13}$, los documentos acerca de las Características de la educación de la Compañía de Jesús ${ }^{14}$, de la pedagogía ignaciana y del paradigma educativo seguido en las instituciones educativas de la Compañía.

b. A partir de este análisis, se efectuaron entrevistas en profundidad con los responsables de la gestión institucional del período de estudio (rector y vicerrectores de la universidad, referentes de la gestión en el contexto del área de Proyección Social de la universidad y responsables de las áreas involucradas en el Vicerrectorado de Medio Universitario) a los fines de caracterizar la PPS, establecer los objetivos buscados del proceso formativo y delimitar las categorías, dimensiones y propiedades, tanto objetivas como perceptuales, a indagar en los graduados para en una etapa posterior, avanzar en la investigación del impacto formativo bajo el paradigma educativo ignaciano.

\section{Resultados y discusiones}

Los resultados que se presentan a continuación son consecuencia de la realización de las dos etapas presentadas, a partir de las cuales es posible exponer la caracterización de la PPS en base a la investigación documental y las entrevistas efectuadas.

La comprensión del Paradigma Pedagógico Ignaciano debe considerar tanto el contexto del aprendizaje como el proceso más explícitamente pedagógico, considerando no sólo el espacio formativo durante el cursado, sino cuando ésta finalice. La PPS desde sus objetivos, contribuye con la pedagogía ignaciana para que las dimensiones que la caracterizan, de contexto, experiencia, reflexión, acción y evaluación puedan darse a lo largo del período de formación y perduren en el tiempo una vez finalizada la carrera. La PPS propicia efectos hacia el interior de la universidad, como también en el medio externo. Este impacto alcanza en el seno de la comunidad universitaria al alumno como principal destinatario, pero también a docentes, personal afectado a la gestión, tareas administrativas y a otros miembros de la comunidad universitaria, puesto la iniciativa en su concreción reclama el 
aporte de diferentes actores a los fines de alcanzar una respuesta de calidad. Por otra parte, una asociación con la comunidad externa y su entorno, precisando los alcances deseados del vínculo, los logros esperados y el tiempo oportuno para alcanzarlos. También se plantean estándares de calidad para la asociación y para la transformación de la realidad fruto de la iniciativa de proyección social, propiciando soluciones integrales y logros a nivel de desarrollo humano, lo cual dista de meros aportes a situaciones coyunturales $\mathrm{o}$ asistemáticas.

Es por ello tan necesario preguntarse y discernir en cada momento histórico cuál es el papel transformador que puede ejercer la universidad en la realidad social concreta en la que se encuentra. La universidad puede adquirir de este modo una determinada proyección social, por medio de la cual ha de "convertirse en una fuerza cultural, que defiende y promueve la verdad, la virtud, el desarrollo y la paz en esa sociedad"15.

La docencia y la investigación son dos tareas esenciales y medulares para cualquier institución de educación superior, que, a través de esta política, no se plantean de manera desarticulada con una tercera función esencial (por propia decisión institucional): la proyección social. La generación del conocimiento y su transmisión poseen un sentido de trascendencia mayor cuando pueden ponerse al servicio de la búsqueda de soluciones para dar respuesta a necesidades sentidas a nivel comunitario. Es de notar que se intenta garantizar desde lo enunciativo el logro de este objetivo, con alcances que beneficien tanto a los alumnos como a la comunidad externa. Y en el caso de los estudiantes, se centra la atención en dos dimensiones de formación: personal y académica. En cuanto al primer aspecto, la formación personal de los estudiantes, es de vital importancia la vivencia de experiencias en contextos de vulnerabilidad social. El abismo de la desigualdad en la que se encuentran muchos de los alumnos, impide que los que mejor viven puedan tener experiencia de la pobreza en la que se halla sumida gran parte de la población mundial y de nuestro país. Sin embargo, es necesario asomarse a esas realidades de pobreza y exclusión para caer en la cuenta de su existencia y así, cuestionarlas vitalmente y con reflexión crítica.

De ahí la importancia de que los alumnos dispongan de experiencias de contacto y servicio a comunidades vulnerables, para conocer su realidad, no solo de forma teórica, sino vital. La transformación ética y de valores se nutre del hecho fundante de salir de uno mismo, reconocer al otro y afirmarlo como persona. La tradición ignaciana enseña que ningún cambio sustancial sucede en la persona si no hay una transformación de la propia sensibilidad. Son este tipo de experiencias las que pueden contribuir a este cambio profundo de la orientación del alumno. "La implicación personal en el sufrimiento inocente, en la injusticia que otros sufren, es el catalizador para la solidaridad que abre el camino a la búsqueda intelectual y a la reflexión moral" 9 .

Un cuestionamiento frecuente es por qué concentrar esfuerzos en poblaciones vulnerables. Promover la justicia conlleva una preocupación primaria por los pobres, defendiendo sus derechos y reorientando hacia ellos los flujos de bienes materiales, culturales, espirituales que benefician de modo constante y preferente a las minorías privilegiadas. La educación universitaria puede contribuir a este proceso de defensa de los pobres, o, por el contrario, ser un instrumento más de los procesos de crecimiento de la desigualdad, pues siempre corre el "riesgo de concentración del saber, exclusión de los débiles y agrandamiento de las diferencias".

La universidad es consciente que este tipo de experiencias corre el riesgo de transformarse en excursiones a la pobreza o convertirse en una clase de turismo social en el que el contacto no vaya más allá de una actividad anecdótica sin consecuencias para la persona. En ocasiones puede convertirse en una más de las numerosas experiencias que el estudiante consume. Es necesario tener en cuenta este riesgo para afrontarlo en la práctica. Se debe tener en cuenta que una verdadera experiencia que pueda contribuir a la formación personal y humana de los estudiantes sucede cuando es sostenida en el tiempo, tal como se enuncia en la política de proyección social de la UCC.

Para no correr este riesgo, las iniciativas deben desarrollarse bajo algunas condiciones: en primer lugar, deben integrarse en la propuesta académica. Para ello deberán estar supervisados por profesores experimentados que dispongan de los conocimientos necesarios en las áreas en las que trabajen los estudiantes, no sólo para capitalizar un aprendizaje disciplinar de calidad, sino para enseñar a aprender en contextos variables, nutrirse de la realidad y acercarlos a la futura vida $\mathrm{y}$ responsabilidad profesional.

En segundo lugar, las experiencias de proyección social deben animar a los estudiantes a compartir sus vivencias, las dificultades que encuentran y los cuestionamientos vitales que afrontan a través de espacios para la reflexión conjunta. Es probable y deseable que algunos alumnos comiencen de forma espontánea procesos de discernimiento sobre su futura orientación profesional. En esas circunstancias es de gran ayuda que haya personas preparadas y capaces de acompañarlos.

También es preciso que los alumnos ejerciten su capacidad para la lectura del propio mundo interior con sus sentimientos y emociones, una práctica característica de la espiritualidad ignaciana. Por

Revista Methodo: Investigación Aplicada a las Ciencias Biológicas. Universidad Católica de Córdoba. Jacinto 
ello, conviene que los servicios a comunidades desfavorecidas incorporen tanto una dimensión profesional, como otra de encuentro humano, no menos importante. Los estudiantes pueden ofrecer sus aún precarios conocimientos profesionales a la comunidad a la que son enviados, sintiéndose útiles y comprobando el valor de sus estudios en el servicio a los demás desde un contexto situado: un "aquí" con sus circunstancias particulares y un "ahora", en la contemporaneidad de la existencia. Es también el modo de no instrumentalizar a los pobres o a poblaciones vulnerables, garantizando el encuentro humano que cuestiona y desencadena procesos interiores.

Un rasgo distintivo que plantea la política de proyección social, es la transformación de la realidad a través de la búsqueda y promoción de soluciones integrales que sean sustentables en el tiempo. Esto se propicia planteando cuidadosamente iniciativas que permitan a través de su planificación, ejecución y consecuencias, resultados de esta clase, y si esto no fuera posible, espacios de reflexión por parte de los estudiantes que posibiliten el análisis de la realidad que los rodea para encontrar las explicaciones que describan y fundamenten las dificultades de éxito según las variables del entorno que los rodea. Es así que el componente de reflexión es indispensable: los estudiantes necesitan estudiar cuáles son las causas que generan las situaciones humanas en las que sirven y cuestionarse sobre el papel que desempeñan los entramados políticos y económicos existentes. Las realidades de injusticia tienden a pasar desapercibidas y la pobreza suele atribuirse a las incapacidades individuales de las personas para salir de su situación. Es necesario estudiar el contexto histórico y cultural, los factores económicos y sociales, las condiciones políticas, para conocer la complejidad de las tramas de la injusticia. La solidaridad universitaria se caracteriza por un serio trabajo intelectual que va de la mano junto a la acción, a través de iniciativas de intervención.

Como señalaba el P. Nicolás ${ }^{15}$, "un reto importante del apostolado intelectual de nuestras universidades proviene hoy del hecho de que la globalización ha creado «sociedades del conocimiento», en las cuales el desarrollo de las personas, culturas y sociedades depende estrechamente del acceso al conocimiento para poder crecer. La globalización ha creado nuevas desigualdades entre los que gozan del poder que les ha dado ese conocimiento, y los que están excluidos de sus beneficios porque no tienen acceso a él".

Las universidades deben reflexionar cuáles son los medios a través de los cuales pueden favorecer la equidad social. Uno de ellos consiste en promover que sus graduados dispongan de una mentalidad y compromiso por la justicia social y por los pobres que les lleve a trabajar por su dignidad y promoción. Parte del éxito de la universidad en el campo de la inclusión social dependerá de las decisiones que estas personas tomen en su vida profesional futura. De ahí la importancia de abrirles a un humanismo cristiano que aspire a un proyecto de dignidad de vida para todas las personas, mediante una educación que tenga una "perspectiva de justicia, desde las necesidades y las esperanzas de los pobres"6.

Otra dimensión a considerar de acuerdo a los objetivos enunciados en la política de proyección social, es el papel de la universidad en la formulación de políticas públicas a nivel social. Las sociedades demandan espacios donde reflexionar sobre sí mismas, lugares de intercambio de ideas, de debate abierto y sincero, de exposición de nuevos modelos de sociedad. Espacios en los cuales se pueda trazar un puente de diálogo entre posturas encontradas, pero sustentables racionalmente. La universidad es uno de estos espacios, para lo cual deben estar abiertos a distintas racionalidades, y conscientes de su influencia cada vez que se expide en determinada materia.

La universidad puede servirse en este espacio para difundir ofertas de sentido, de vida digna y de solidaridad de un modo preferente. Puede hacer en él presente la voz de los últimos en comunidades en las que sus consideraciones y percepciones de la realidad están ausentes. Esta voz debe ser rigurosa y bien sustentada, apoyada en los valores que se sustentan, y dialogando con los sectores sociales, sean públicos o privados.

Como se expresa en el documento La Promoción de la Justicia en las Universidades de la Compañía ${ }^{16}$, es también el lugar desde el que lanzar las propuestas de vida y de justicia que surgen de la propia reflexión universitaria. Se trata por tanto de un ágora pública desde la que entrar en diálogo con la cultura actual. Un espacio de formación permanente para la propia sociedad local: "La universidad debe tener la valentía de expresar verdades incómodas... para salvaguardar el bien auténtico de la sociedad" ". Es uno de los modos en que se puede reconocer la identidad de la propia obra.

Cuando esto sucede y la universidad toma un posicionamiento público, se ubica en una posición de tensión frente a las relaciones que ha establecido con los poderes políticos, sociales y económicos, que expresarán su acuerdo o su malestar. En ocasiones, esos poderes pueden estar representados en los propios órganos de la universidad, ejerciendo presión para que se tomen o no determinadas posturas. "Puede suceder que no todos los patronos o miembros de los consejos de gobierno sean siempre desinteresados, ni se 
identifiquen necesariamente con las declaraciones de misión y con la orientación de la universidad... La institución acabará por moderar el tono de su voz, o tendrá que renunciar a hablar de ciertos asuntos" $"$. Entonces se ponen a prueba las motivaciones últimas de la propia universidad, pues debe decidir cuáles son los valores que antepone.

Una vez realizado el análisis documental sobre las bases en las que se sustenta la pedagogía ignaciana seguida en todas las instituciones educativas confiadas a la Compañía de Jesús, cuya síntesis se ha presentado, se efectuaron entrevistas en profundidad con referentes institucionales de la Universidad Católica de Córdoba a los fines de contrastar la propia realidad local en la ejecución no sólo de la PPS, sino del proceso educativo integral que se lleva a la práctica en correlación con lo enunciado en los principios de la pedagogía ignaciana.

A través de las entrevistas efectuadas fue posible trazar con foco situado en los graduados de esta universidad, los aspectos o características deseadas como consecuencia del proceso formativo tras la implementación de la PPS y la vivencia de la pedagogía ignaciana.

Es notable la gran correlación existente entre los entrevistados acerca de los logros deseados en la formación de los graduados.

Ante esta pregunta central, los entrevistados expresaron las siguientes consideraciones presentadas en (Tabla 1), en total sincronía con lo que es posible indagar en la investigación documental sobre las fuentes consultadas acerca del proceso pedagógico ignaciano:

A modo de síntesis, y tras los elementos presentados, es posible interrelacionar los hallazgos a través de la investigación documental, los testimonios de los referentes institucionales y lo establecido a través de la PPS, cuyo impacto se pretende medir.

En este sentido, el abordaje del concepto de proyección social adoptado por la universidad y los objetivos propuestos a través de la PPS institucional a través de su caracterización, hacen posible comprender en primera instancia que los efectos de su aplicación buscan alcanzar objetivos desde dos dimensiones:

A. Por un lado, el modo en que se relaciona la Universidad con la comunidad y su entorno o zona de influencia, a través de la PPS. Se pretende que la participación institucional en relación a la comunidad sea de calidad, con un involucramiento activo de quienes participan de la experiencia, a través de actividades concretas y directas, logrando una contribución real dirigida preferentemente hacia los sectores más desfavorecidos del entramado social. Se establece una relación o asociación ente la universidad y la comunidad en torno a la coproducción de saberes, donde el conocimiento es dinámico y bidireccional entre las partes, a través de la creación de una comunidad de aprendizaje, multidisciplinaria en función de las potencialidades de las distintas áreas de la universidad logrando una sinergia institucional para que a través de la academia se logren aportar soluciones a necesidades sentidas a nivel social y comunitario (Tabla 2).

B. La segunda dimensión se relaciona con los efectos esperados sobre los alumnos (futuros graduados) a través de la ejecución de la PPS:

1.Acciones de impacto externo, hacia la comunidad o zona de influencia donde ejerce su profesión.

2.Efectos hacia el interior de la persona a medida que el proceso de formación avanza y los elementos que caracterizan el paradigma ignaciano se implementan a través de la experiencia, la reflexión y la acción en un contexto situado.

Es necesario observar, además, que estos impactos pueden ser sujetos de medición de acuerdo a las dimensiones y propiedades definidas, de manera objetiva o perceptual dependiendo del atributo a considerar.

En la (Tabla 3) se exponen los resultados de esta caracterización.

\section{Conclusiones}

Los resultados de caracterización de la PPS permiten considerar dos categorías de análisis: por un lado, el vínculo de la Universidad con la comunidad de acuerdo a propiedades prestablecidas, y por otro, el impacto de la política sobre los futuros graduados de acuerdo al paradigma educativo adoptado. Esta segunda consideración involucra un impacto hacia el entorno, con consecuencias a nivel socio ambiental y en torno a políticas públicas; y un impacto interno, con efectos hacia la formación personal y humana, la formación académica y profesional, y aspectos relacionados con la transformación interna de la propia persona.

La política de proyección social adoptada por la UCC se sustenta en un modelo educativo, cuyo impacto en la formación de los graduados, es un insumo fundamental para la autoevaluación institucional de esta universidad, y de todas las confiadas a la Compañía de Jesús.

La caracterización de la PPS institucional presentada aporta elementos necesarios a los fines de avanzar en la medición de este impacto, contribuyendo al proceso de institucionalización de la Responsabilidad Social Universitaria y a

Revista Methodo: Investigación Aplicada a las Ciencias Biológicas. Universidad Católica de Córdoba. Jacinto

Ríos 571 Bo Gral. Paz. X5004FXS. Córdoba. Argentina. Tel.: (54) 3514517299 / Correo: methodo@ucc.edu.ar

/ Web: methodo.ucc.edu.ar | ARTICULO DE REVISION Rev. Methodo 2021;6 (1):33-43. 
afianzar el paradigma educativo en la búsqueda de formar personas competentes, conscientes, compasivas y comprometidas en el ejercicio de la profesión.

La investigación propone aportar en etapas posteriores, herramientas metodológicas que permitan correlacionar los procesos educativos de acuerdo a la política de proyección social planteada en la universidad con respecto a la formación y perfil de sus graduados.

La UCC carece de esta herramienta, por lo cual podría resultarle de utilidad para completar su proceso de autoevaluación y avanzar en la medición de la efectividad de su política de proyección social, al tiempo que hacerla extensiva a otras universidades jesuitas de América Latina.

\section{Bibliografía}

1. Asociación de Universidades Jesuitas de Latinoamérica (AUSJAL). Políticas y sistema de autoevaluación y gestión de la Responsabilidad Social Universitaria en AUSJAL. Alejandría.2009.

2. Resolución Rectoral $\mathrm{N}^{\circ} 1092 / 2011$. Política relativa a la proyección social en la Universidad Católica de Córdoba. 2011. [Consultado 22 de agosto de 2020]. Disponible en Digesto de la Universidad Católica de Córdoba. https://bit.ly/2QLyVBq

3. Ugalde, L. Conscientes, competentes, compasivos y comprometidos. 2012. [Consultado 22 de agosto de 2020]. Disponible en https://www.ausjal.org/wpcontent/uploads/Conscientes2C-competentescompasvios-y-comprometidos.pdf.

4. Kolvenbach, P.H. La Universidad jesuita hoy. En Documentes Corporativos I. Compañía de Jesús Apostolado Educativo. 1985. p 472- 482.

5. Kolvenbach, P. H. En el segundo centenario de la enseñanza jesuítica en Estados Unidos de América. En Selección de escritos del P. Peter-Hans Kolvenbach, 1983 - 1990. 1989. p 400 - 409.

6. Kolvenbach, P. H. A la universidad iberoamericana sobre un nuevo modelo de universidad. En Selección de escritos del P. Peter-Hans Kolvenbach, 1983 - 1990. 1990. p $410-417$.

7. Kolvenbach, P. H. Discurso con motivo de la celebración del Primer Centenario de la
Universidad Pontificia Comillas. En Selección de escritos del P. Peter-Hans Kolvenbach, 1991 - 2007. 1991. p 260 - 271.

8. Kolvenbach, P. H. Alocución en la Universidad de Saint Joseph. En Selección de escritos del P. Peter-Hans Kolvenbach, 1991 2007. 2000a. p 281 - 293.

9. Kolvenbach, P. H. El servicio de la fe y la promoción de la justicia en la educación universitaria de la Compañía de Jesús en los Estados Unidos. En Selección de escritos del P. Peter-Hans Kolvenbach, 1991 - 2007. 2000b. p 294 - 310.

10. Kolvenbach, P. H. La Compañía de Jesús a la luz del carisma ignaciano. En Selección de escritos del P. Peter-Hans Kolvenbach, 1991 2007. 2001. p 311 - 326.

11. 11. Congregación General XXXII de la Compañía de Jesús. Decreto $4^{\circ}$. nuestra misión hoy: el servicio a la fe y la promoción de la justicia. Razón y Fe. 1975.

12. Arrupe P. Alocución al X Congreso de la Confederación Europea de Asociaciones de Antiguos Alumnos de Jesuitas. en Hombres para los demás. Diafora 1973. p 159.

13. Arrupe P. La promoción de la Justicia y la formación en las Asociaciones de Antiguos Alumnos. En Iglesia y Justicia. Actas del X Congreso de la Confederación Europea de Asociaciones de AA. de Jesuitas. 1973.

14. Características de la Educación de la Compañía de Jesús. (1986). Comisión Nacional de Educación. 1986.

15. Nicolás, A. Profundidad, universalidad y ministerio intelectual. Retos para la educación superior jesuita hoy. En Redes para la Educación Superior Jesuita: configurar un futuro para un mundo humano, justo, $\mathrm{y}$ sostenible. 2010.

Secretariado para la Justicia Social y Ecológica. La Promoción de la Justicia en las Universidades de la Compañía (de Jesús). Promotio Iustitiae, 2014. 116. Disponible en: https://www.sjesjesuits.global/wpcontent/uploads/PJ_116_ESP.pdf

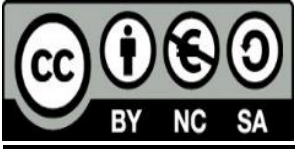




\section{Anexo tablas}

Tabla 1. Atributos buscados en la formación de los graduados de la UCC señalados por los referentes institucionales según guion validado para las entrevistas en profundidad efectuadas.

\begin{tabular}{|c|c|}
\hline \multirow{2}{*}{\multicolumn{2}{|c|}{ Posicionamiento ético respecto a la profesión. }} \\
\hline & \\
\hline & $\begin{array}{l}\text { Responsabilidad con respecto a los problemas sociales (como individuo y como } \\
\text { parte de la sociedad). }\end{array}$ \\
\hline & $\begin{array}{l}\text { Graduados con Ciencia (competentes), Conciencia (ética en su desempeño), } \\
\text { Compromiso (no sólo personas éticas, sino que hagan una diferencia a través de } \\
\text { las acciones) y compasión hacia los más desfavorecidos. }\end{array}$ \\
\hline & La importancia de los graduados radica en el bien que puedan hacer a la sociedad. \\
\hline & $\begin{array}{l}\text { Que sean agentes de transformación o agentes de cambio, para que desde su lugar } \\
\text { pueden dar el mayor servicio posible a su entorno. }\end{array}$ \\
\hline \multirow{4}{*}{ 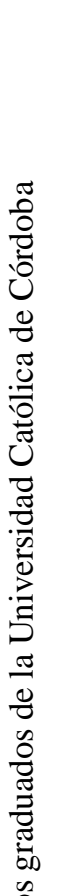 } & $\begin{array}{l}\text { Se busca formar personas sólidas, críticas, compasivas, comprometidas, útiles y } \\
\text { prácticas, coherentes entre lo que dicen y hacen. }\end{array}$ \\
\hline & Se busca formar profesionales que propicien un modelo de sociedad diferente. \\
\hline & Se busca formar agentes de transformación de las estructuras. \\
\hline & Un egresado que sienta interpelado y actúe desde lo que es. \\
\hline \multirow{9}{*}{ 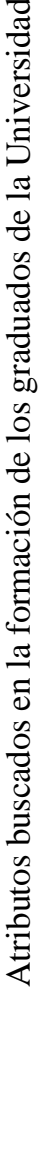 } & Que sientan preocupación por la justicia e incomodidad por una buena causa. \\
\hline & $\begin{array}{l}\text { Que sean líderes positivos y que ayuden a ser buenas o mejores personas los que } \\
\text { están en su entorno. }\end{array}$ \\
\hline & Que vean que el servicio profesional mejora la vida de las demás personas. \\
\hline & $\begin{array}{l}\text { Que sean valientes en el ejercicio para poner en práctica la ética en la vida } \\
\text { profesional. }\end{array}$ \\
\hline & $\begin{array}{l}\text { Que en el ejercicio no pongan en primer lugar criterios cómodos como el ganar } \\
\text { dinero, sino criterios basados donde haya mayor necesidad de sus aportes. }\end{array}$ \\
\hline & Que manifiesten una coherencia puesta en práctica. \\
\hline & Que generen espacios positivos para colaborar. \\
\hline & Que posean una formación en valores e integral. \\
\hline & Que desarrollen competencias transversales que van más allá de lo disciplinar. \\
\hline
\end{tabular}


Tabla 2. Características del vínculo Universidad-Comunidad a través de la PPS.

\begin{tabular}{|c|c|c|}
\hline Categoría & Subcategorías & Dimensiones \\
\hline \multirow{12}{*}{$\begin{array}{c}\text { Vínculo Universidad- } \\
\text { Comunidad }\end{array}$} & \multirow{6}{*}{ Participación } & De calidad \\
\hline & & Con involucramiento activo \\
\hline & & Actividades concretas y directas \\
\hline & & Contribución real \\
\hline & & Preferencia por sectores desfavorecidos. \\
\hline & & Resolución de problemas \\
\hline & \multirow{6}{*}{$\begin{array}{c}\text { Coproducción de } \\
\text { saberes }\end{array}$} & Asociación Universidad-Comunidad \\
\hline & & Conocimiento bidireccional \\
\hline & & Intersectorialidad \\
\hline & & Comunidad de aprendizaje \\
\hline & & Multidisciplinar \\
\hline & & Sinergia institucional \\
\hline
\end{tabular}

Fuente: elaboración propia (2020).

Tabla 3. Impactos esperados de la PPS sobre los graduados de la UCC.

\begin{tabular}{|c|c|c|c|c|}
\hline Categorías & Subcategorías & Dimensiones & $\begin{array}{l}\text { Propiedades } \\
\text { objetivas a medir } \\
\text { sobre los } \\
\text { graduados }\end{array}$ & $\begin{array}{l}\text { Propiedades perceptuales a } \\
\text { medir sobre los graduados }\end{array}$ \\
\hline & & $\begin{array}{l}\text { Cualidades } \\
\text { ciudadanas }\end{array}$ & & $\begin{array}{l}\text { Cultura tributaria y } \\
\text { cumplimiento de deberes } \\
\text { laborales. } \\
\text { Influencia de la PPS y } \\
\text { formación UCC en este } \\
\text { aspecto. }\end{array}$ \\
\hline $\begin{array}{l}\text { Impacto } \\
\text { Externo }\end{array}$ & $\begin{array}{l}\text { Transformació } \\
\text { n de la realidad } \\
\text { socio } \\
\text { ambiental }\end{array}$ & $\begin{array}{l}\text { Compromiso } \\
\text { socio } \\
\text { ambiental }\end{array}$ & $\begin{array}{l}\text { Acciones o } \\
\text { proyectos sociales, } \\
\text { sustentables } \\
\text { promovidos. } \\
\text { Acciones realizadas } \\
\text { a favor de la } \\
\text { Promoción de la } \\
\text { Justicia entendida } \\
\text { desde la formación } \\
\text { de la UCC y su PPS }\end{array}$ & $\begin{array}{l}\text { Soluciones integrales logradas. } \\
\text { Autopercepción } \\
\text { compromiso social } \\
\text { medioambiental. } \\
\text { Significado personal de la } \\
\text { palabra "justicia". Comprensión } \\
\text { del concepto de Promoción de la } \\
\text { Justicia explicitado en la PPS. } \\
\text { Autopercepción de actor y líder. } \\
\text { Influencia de la formación }\end{array}$ \\
\hline
\end{tabular}




\begin{tabular}{|c|c|c|c|c|}
\hline & & & $\begin{array}{l}\text { Acciones a favor del } \\
\text { medioambiente }\end{array}$ & $\begin{array}{l}\text { universitaria en las acciones } \\
\text { vinculadas al compromiso socio } \\
\text { ambiental. }\end{array}$ \\
\hline & & $\begin{array}{l}\text { Ejercicio } \\
\text { ético de la } \\
\text { profesión }\end{array}$ & $\begin{array}{l}\text { Ausencia de } \\
\text { Sanciones } \\
\text { deontológicas }\end{array}$ & $\begin{array}{l}\text { Cumplimiento del Código de } \\
\text { Ética de ejercicio profesional. } \\
\text { Concepción ética del ejercicio } \\
\text { profesional }\end{array}$ \\
\hline & $\begin{array}{l}\text { Incidencia en } \\
\text { políticas } \\
\text { públicas }\end{array}$ & $\begin{array}{l}\text { Participación } \\
\text { y/o aportes a } \\
\text { las políticas } \\
\text { públicas }\end{array}$ & $\begin{array}{l}\text { Cargos de liderazgo } \\
\text { desempeñados en } \\
\text { instituciones } \\
\text { estatales o privadas } \\
\text { de bien público }\end{array}$ & $\begin{array}{l}\text { Aportes que hayan incidido en } \\
\text { la agenda de instituciones } \\
\text { estatales o privadas de bien } \\
\text { público. } \\
\text { Reflexión personal sobre la } \\
\text { importancia de la participación } \\
\text { en políticas públicas }\end{array}$ \\
\hline \multirow{7}{*}{$\begin{array}{l}\text { Impacto } \\
\text { Interno }\end{array}$} & \multirow{4}{*}{$\begin{array}{l}\text { Formación } \\
\text { personal/ } \\
\text { humana }\end{array}$} & \multirow{2}{*}{$\begin{array}{l}\text { Formación } \\
\text { en valores }\end{array}$} & & $\begin{array}{l}\text { Escala de valores personales. } \\
\text { Concepción asociada a la noción } \\
\text { de "buena persona" }\end{array}$ \\
\hline & & & & $\begin{array}{l}\text { Escala de Valores } \\
\text { Profesionales. } \\
\text { Concepción asociada a la noción } \\
\text { de "profesional exitoso" }\end{array}$ \\
\hline & & \multirow{2}{*}{$\begin{array}{l}\text { Reconoci- } \\
\text { miento } \\
\text { crítico de la } \\
\text { realidad y de } \\
\text { la profesión }\end{array}$} & & $\begin{array}{l}\text { Problemáticas sociales } \\
\text { vinculadas a la profesión } \\
\text { visualizadas sujetas de } \\
\text { atención }\end{array}$ \\
\hline & & & & $\begin{array}{l}\text { Problemáticas sociales } \\
\text { vinculadas a la profesión } \\
\text { factibles de ser resueltas desde } \\
\text { su ejercicio profesional }\end{array}$ \\
\hline & \multirow{2}{*}{$\begin{array}{l}\text { Formación } \\
\text { académica y } \\
\text { profesional }\end{array}$} & \multirow{2}{*}{$\begin{array}{l}\text { Competencia } \\
\text { disciplinar }\end{array}$} & \multirow{2}{*}{$\begin{array}{l}\text { Promedio de egreso } \\
\text { y años de cursado }\end{array}$} & $\begin{array}{l}\text { Consideración personal de su } \\
\text { preparación/competencia } \\
\text { académica/profesional. }\end{array}$ \\
\hline & & & & $\begin{array}{l}\text { Nivel de inserción laboral } \\
\text { (tiempo/cargo/institución) }\end{array}$ \\
\hline & $\begin{array}{l}\text { Transformació } \\
\text { n interna }\end{array}$ & $\begin{array}{l}\text { Experiencia } \\
\text { vivencial }\end{array}$ & $\begin{array}{l}\text { Participación } \\
\text { durante su período } \\
\text { formativo en }\end{array}$ & $\begin{array}{l}\text { Incidencia que esta } \\
\text { participación ha tenido en las } \\
\text { futuras elecciones/opciones }\end{array}$ \\
\hline
\end{tabular}




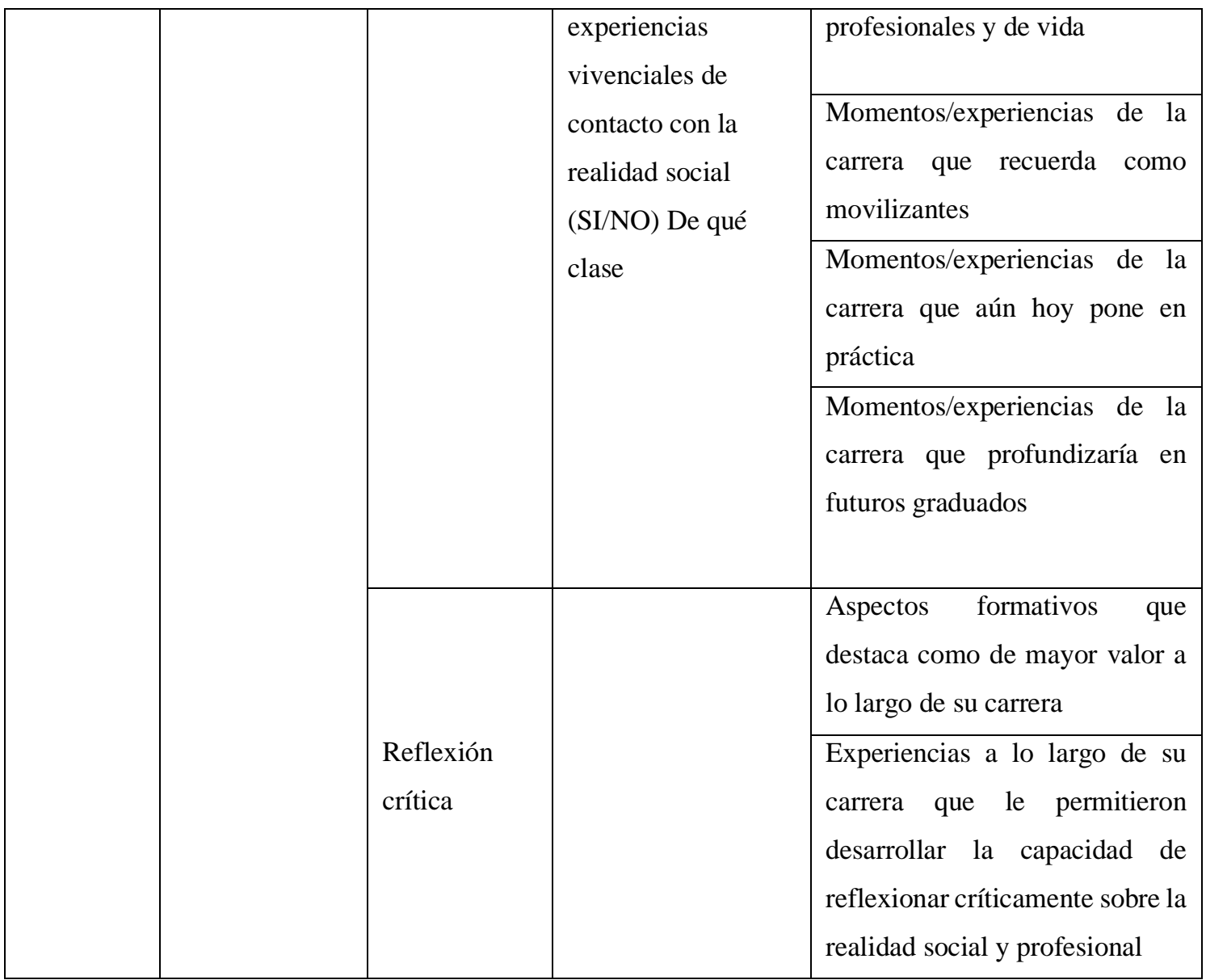

Fuente: elaboración propia (2020).

\section{(c) (1) \&(0)

